\title{
Late Presentation of an Inferior Myocardial Infarction Complicated by Ventricular Septal Rupture: A Case Report
}

\author{
Weixian Alex Tan ${ }^{a}$,, Yi Kit Philip Panga, Yeow Leng Chua ${ }^{a}$, \\ Sung Lung Aaron Wong ${ }^{\mathrm{a}}$
}

\begin{abstract}
Ventricular septal rupture (VSR) is a well-described and potentially fatal complication of acute myocardial infarctions. In the era of early reperfusion therapy, the incidence has been on the decline. We describe a case of delayed presentation inferior myocardial infarction complicated by VSR requiring early surgery and review our center's experience.
\end{abstract}

Keywords: Inferior myocardial infarction; Ventricular septal rupture; Complication; Surgery

\section{Introduction}

Ventricular septal rupture (VSR) is a well-known and potentially fatal complication following acute myocardial infarction. In the pre-thrombolytic era, the incidence was $1-2 \%$ but has since declined to $0.17-0.31 \%$ with the advent of reperfusion therapy [1]. VSRs have been reported to occur $16-24$ $\mathrm{h}$ after acute myocardial infarction in the reperfusion era as opposed to 3 - 5 days previously [1,2]. The hypothesis for earlier development of VSR is due to reperfusion injury and fibrinolysis [1].

VSR should be suspected clinically in patients with recent myocardial infarction who present with signs of cardiac failure, a new holosystolic murmur over the precordium. This can be confirmed on echocardiography with color flow Doppler imaging.

\section{Case Report}

A 54-year-old Indonesian male with a 48 pack years smoking

Manuscript accepted for publication October 18, 2016

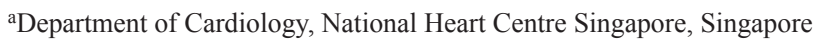
${ }^{b}$ Corresponding Author: Weixian Alex Tan, Department of Cardiology, National Heart Centre Singapore, 5 Hospital Drive, Singapore 169609, Singapore.Email: alex.tan@mohh.com.sg

doi: http://dx.doi.org/10.14740/cr502w history, hypertension, diabetes mellitus, and dyslipidemia saw his local doctor for chest pain and dyspnea. He was treated medically for an inferior ST elevation myocardial infarction.

He presented to our center in Singapore a week later with persistent dyspnea, orthopnea and lower limb swelling. Clinical examination revealed a grade $3 / 6$ holosystolic murmur loudest over the left lower sternal edge, reduced breath sounds bibasally and inspiratory crepitations bilaterally to the mid zone.

Electrocardiogram (ECG) (Fig. 1) showed Q waves in the inferior leads with $2 \mathrm{~mm} \mathrm{ST}$ elevations and T-wave inversions, suggestive of a recent inferior myocardial infarction. Chest Xray (Fig. 2) showed bilateral pleural effusions with pulmonary venous congestion.

Transthoracic echocardiography showed a thinned and aneurysmal inferoseptal and inferior left ventricular wall from basal to mid-level. A VSR was also seen within the aneurysm with left to right shunt (Fig. 3). We proceeded to perform a cardiac MRI which revealed VSR at mid inferoseptal wall (Fig. 4), with a basal to mid inferior wall aneurysm and significant intracardiac shunt (QP/QS 3.3). A diagnostic angiogram was performed which showed triple vessel disease with diffuse stenosis in the LAD and circumflex vessels and $100 \%$ stenosis of the mid RCA (Fig. 5a, b).

After consultation with the cardiothoracic team, early coronary artery bypass graft and VSR repair were planned. However, the patient deteriorated rapidly into cardiogenic shock. Dopamine infusion was started, an intra-aortic balloon pump was inserted and he was brought for urgent operation.

During the operation, the inferior myocardial infarct was evident by hemorrhagic myocardium between the right posterior descending artery (RPDA) and right posterolateral artery (RPL) vessels. The large VSR was next to the tricuspid valve septal attachment rendering the valve incompetent. Coronary artery bypass grafting was performed, saphenous vein graft (SVG) to left anterior descending coronary artery (LAD) and SVG to obtuse marginal branch (OM). Ventriculotomy was made into the infarcted tissue between the RPDA and RPL and patch closure of VSR was performed. The infarcted right inferior ventricular wall was resected and the ventriculotomy was closed. The native tricuspid valve leaflet was excised and a bioprosthetic valve was implanted.

Postoperative course was complicated by oliguric acute kidney injury requiring temporary dialysis. The patient also 


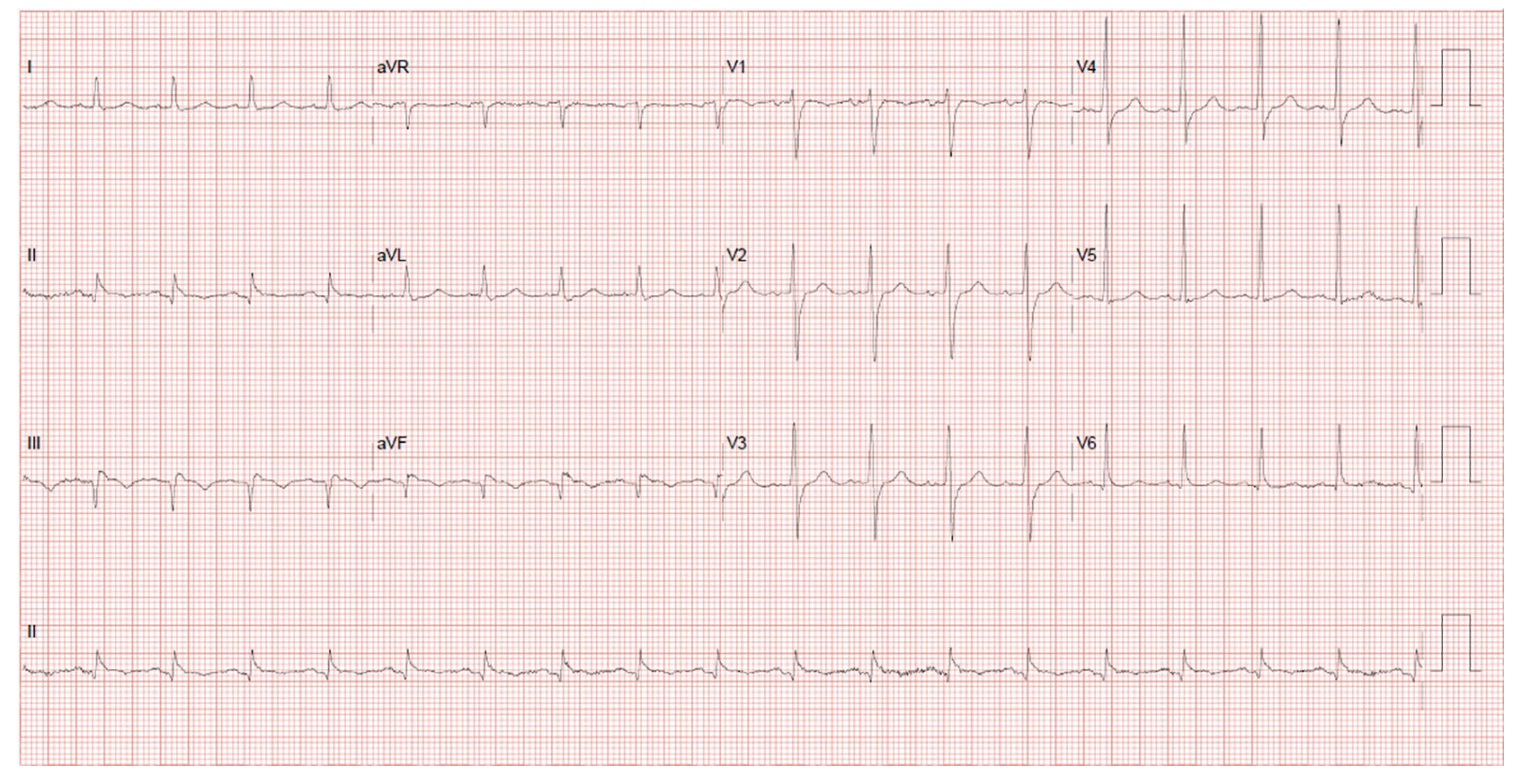

Figure 1. $Q$ waves in the inferior leads with $2 \mathrm{~mm} \mathrm{ST}$ elevations and T-wave inversions, suggestive of a recent inferior myocardial infarction.

developed complete heart block requiring dual chamber permanent pacemaker insertion with right atrial lead and right ventricular lead placed through the coronary sinus. He subsequently made a steady recovery.

\section{Discussion}

This patient is the first in our center's series where there was inadequate healthy margins to place the VSR patch without compromising the tricuspid valve leaflets and conduction pathway, thus necessitating a tricuspid valve replacement. From our surgical series of 47 patients since 2000, we have encountered 13 with inferior VSRs. Mortality rates were higher,

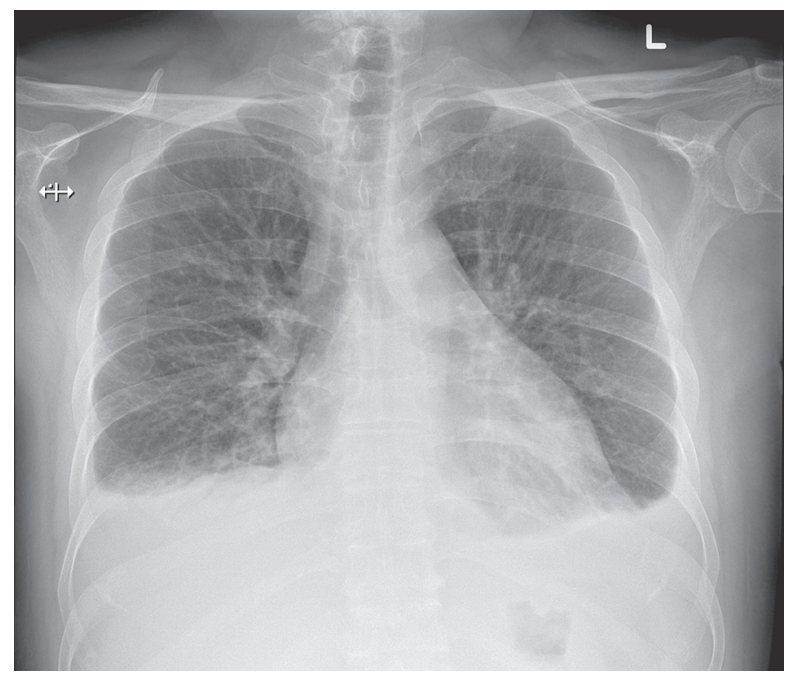

Figure 2. Bilateral pleural effusions with pulmonary venous congestion.

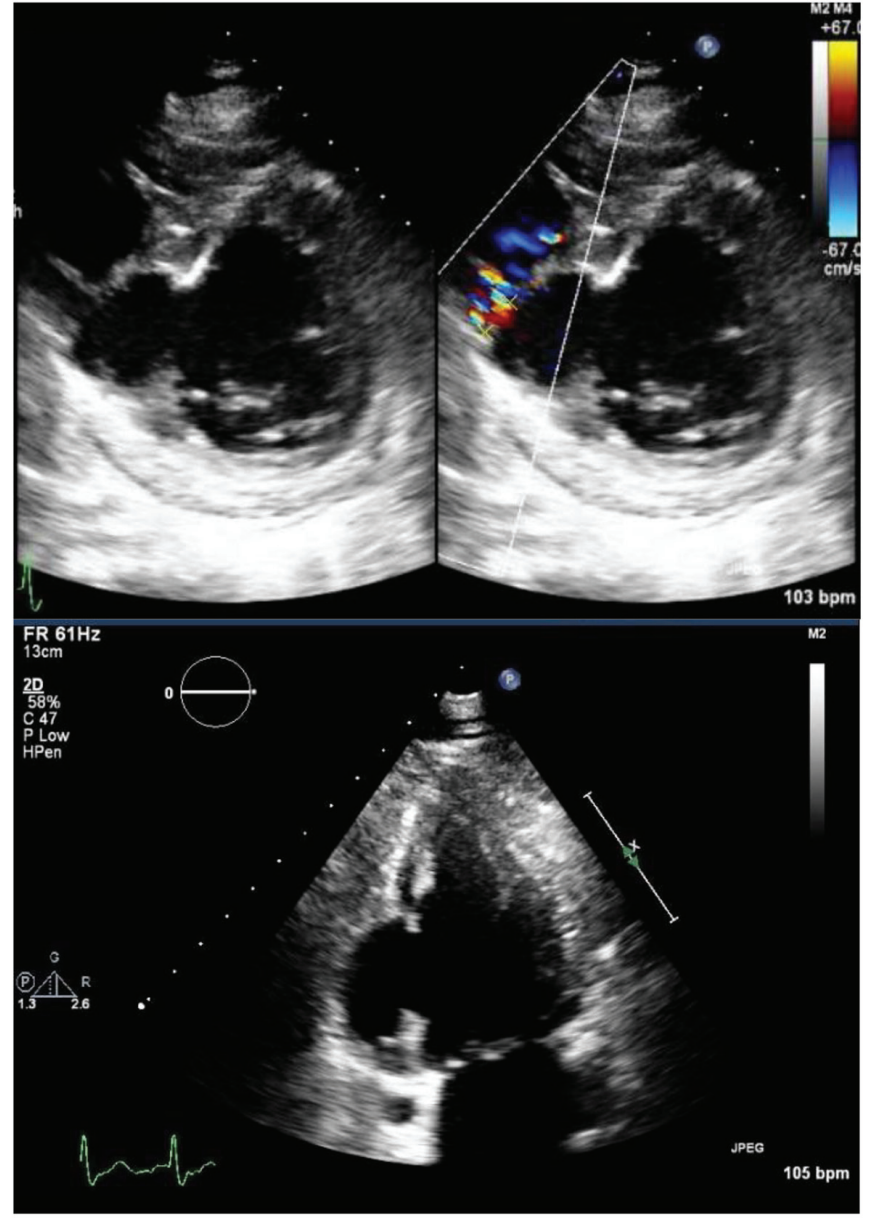

Figure 3. VSR was also seen within the aneurysm with left to right shunt. 


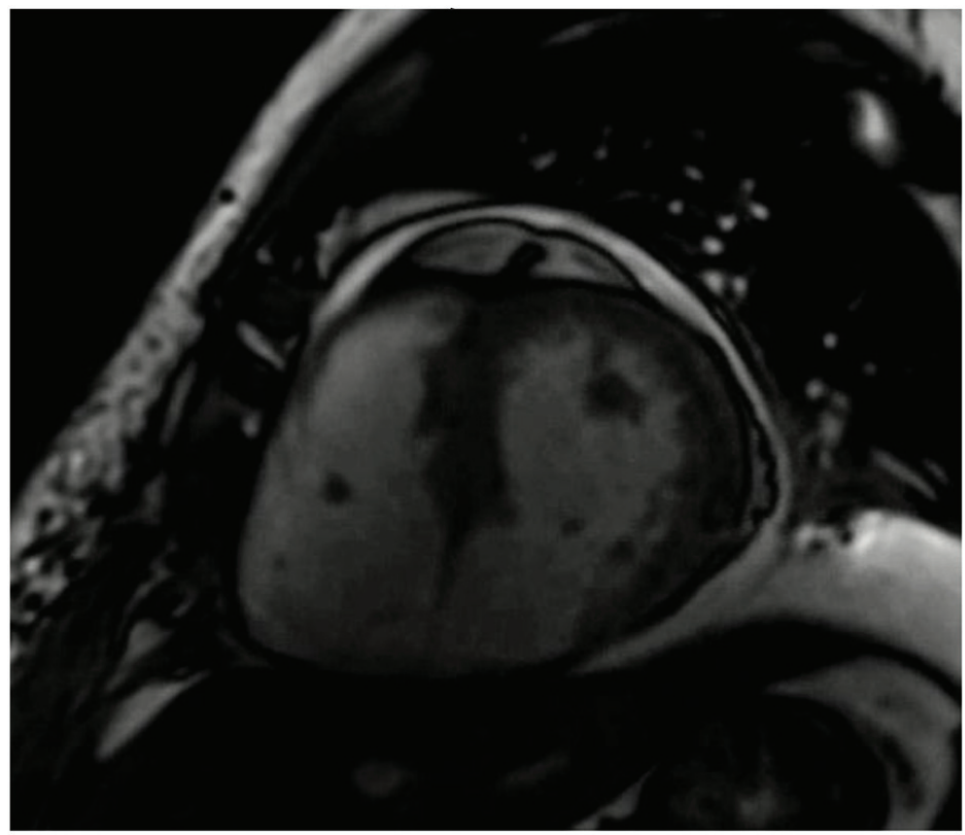

Figure 4. LV short axis view shows VSR is at the mid inferoseptal wall with a basal to mid inferior wall aneurysm and significant intracardiac shunt (QP/QS 3.3).

$7 / 13$ (54\%) for inferior VSRs compared to $11 / 34(32 \%)$ for anterior VSRs. This could be due to a combination of factors such as difficult surgical access, right ventricular dysfunction, increased risk of conduction block and a potentially higher incidence of residual shunts.

It is believed that this is the first VSR repair case that required permanent pacing postoperatively, likely due to the compromised conduction pathway from the infarcted tissue and VSR patch placement. Furthermore, in view of the friable nature of the infarcted right ventricle and bioprosthetic tricuspid valve, the right ventricular lead of the pacemaker had to be inserted via the coronary sinus.

Timing to surgery should be decided on a case to case ba- sis taking into account the hemodynamic stability of the patient $[1,3]$. As reported in the Society of Thoracic Surgeons National Database, improved outcomes were seen with delayed surgery with $54.1 \%$ mortality (surgery within 7 days) vs. $18.4 \%$ (surgery after 7 days) as those requiring early surgery are likely sicker with greater hemodynamic instability $[1,4]$. In our center, only a selected subset (three of 47) of patients in our series were stable enough to undergo surgery more than 2 weeks after diagnosis.

With the advent of early reperfusion strategies, the incidence of VSR has decreased. However, it is still a serious complication that should not be overlooked especially in cases of myocardial infarction which presents late.
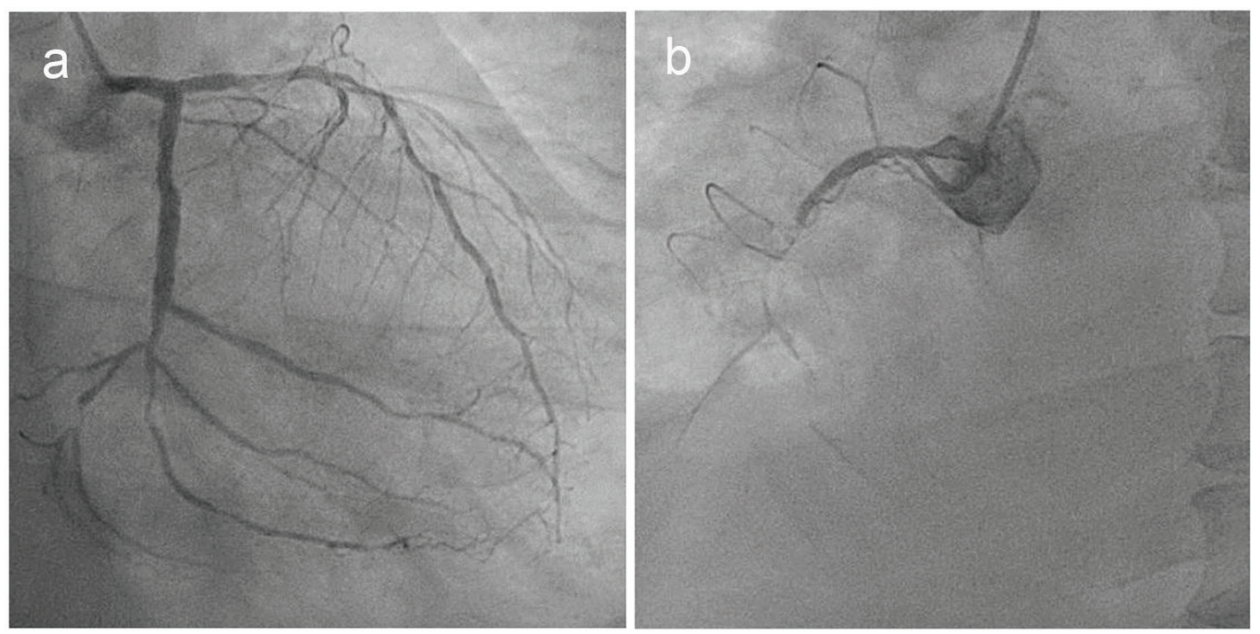

Figure 5. (a) Diffuse stenosis was seen in the left anterior descending and circumflex vessels. (b) $100 \%$ stenosis of the mid right coronary artery was seen. 


\section{Conflicts of Interest}

Authors have nothing to disclose with regard to conflicts of interest to this case report.

\section{Sources of Funding}

No funding was provided for the writing of this case report.

\section{References}

1. Jones BM, Kapadia SR, Smedira NG, Robich M, Tuzcu
EM, Menon V, Krishnaswamy A. Ventricular septal rupture complicating acute myocardial infarction: a contemporary review. Eur Heart J. 2014;35(31):2060-2068.

2. Birnbaum Y, Fishbein MC, Blanche C, Siegel RJ. Ventricular septal rupture after acute myocardial infarction. N Engl J Med. 2002;347(18):1426-1432.

3. Papalexopoulou N, Young CP, Attia RQ. What is the best timing of surgery in patients with post-infarct ventricular septal rupture? Interact Cardiovasc Thorac Surg. 2013;16(2):193-196.

4. Arnaoutakis GJ, Zhao Y, George TJ, Sciortino CM, McCarthy PM, Conte JV. Surgical repair of ventricular septal defect after myocardial infarction: outcomes from the Society of Thoracic Surgeons National Database. Ann Thorac Surg. 2012;94(2):436-443; discussion 443-434. 